\title{
The Non-pneumatic Anti-Shock Garment: How Applier Strength and Body Mass Index Affect External Abdominal Pressure
}

\author{
Amy Stenson ${ }^{1}$, Felicia Lester ${ }^{2}$, Carinne Meyer ${ }^{3}$, Jessica L. Morris ${ }^{*}, 3$, Juan Vargas $^{3}$ and \\ Suellen Miller ${ }^{3}$
}

\author{
${ }^{I}$ Department of Obstetrics and Gynecology, David GeffenSchool of Medicine at the University of California, Los \\ Angeles, California, USA \\ ${ }^{2}$ Department of Obstetrics and Gynecology, Brigham and Women's Hospital, Boston, Massachusetts, USA \\ ${ }^{3}$ Department of Obstetrics, Gynecology and Reproductive Sciences, University of California, San Francisco, California, \\ USA
}

\begin{abstract}
Objective: To assess the amount of abdominal pressure generated by the Non-pneumatic Anti-Shock Garment (NASG), a first-aid counter pressure device for obstetric hemorrhage, and to evaluate if body mass index (BMI) and applier strength, affect pressure.

Study Design: A pilot study convenience sample of 20 non-pregnant volunteers underwent external abdominal pressure monitoring with NASG placement. Mean pressure and the effects of BMI and applier strength were examined.

Results: The mean external abdominal pressure significantly increased from $1.0 \mathrm{mmHg}$ at baseline to $67 \mathrm{mmHg}$ on NASG application, returning to $1.0 \mathrm{mmHg}$ upon removal $(\mathrm{p}=0.005)$. Greater mean pressure was exerted by a strong applier versus a weak applier, irrespective of BMI $(\mathrm{p}<0.001)$. Pressure had an inverse relationship with BMI, particularly with a strong applier $(\mathrm{r}=-0.905, \mathrm{p}<0.001)$. The difference between pressures achieved in an underweight patient with a strong applier and an overweight patient with a weak applier was significant $(73.2 \mathrm{vs} 35.7 \mathrm{mmHg}$; $\mathrm{p}=0.051)$.

Conclusion: This pilot study demonstrates a significant increase in abdominal pressure with device application and significant variation with BMI and applier strength. These findings may have important implications for optimizing device usage.
\end{abstract}

Keywords: Non-pneumatic anti-shock garment, maternal mortality, obstetric hemorrhage, pressure, body mass index, strength.

\section{INTRODUCTION}

Every day 1,000 women die in childbirth $[1,2]$. Globally, severe obstetric hemorrhage is the leading cause of maternal death, largely due to delays in accessing life-saving services such as surgery or blood transfusion [3, 4]. Effective, reliable means of stabilizing patients during delays would save many mothers' lives, which is the premise underlying use of the Non-pneumatic Anti-Shock Garment (NASG) in lowresource settings. The NASG is an articulated neoprene and Velcro ${ }^{\circledR}$ first-aid compression device that was designed to reverse shock in trauma patients by shunting blood from the lower extremities and pelvis to the vital organs (Fig. 1) [5, 6].

Clinical trials into the NASG (Zoex, Coloma, CA 95613) have been conducted in Egypt and Nigeria according to a pre-intervention/post-intervention design. For women treated with the NASG, measured blood loss after entry to the study was reduced from $33-78 \%$ and the NASG improved maternal

*Address correspondence to this author at the Safe Motherhood Program, Department of Obstetrics ,Gynecology and Reproductive Sciences, University of Califormia, San Francisco, 50 Beale Street, Suite 1200, San Francisco , CA 94105, USA; Tel: 4155979343; Fax: 4155979300;

E-mail: jemo307@gmail.com survival by 40-67\% [7-9]. Despite these improved statistics, unexplained maternal morbidity (e.g., anuria, dyspnea) and deaths still occur, even with use of the NASG. Women presenting in shock and placed into the NASG may have already lost significant amounts of blood, resulting in deaths and/or complications. However, it is also possible that adverse maternal outcomes could involve suboptimal application of the NASG. At this time, the cause of the residual maternal morbidity and mortality has not been established and although the NASG could not be expected to result in a $100 \%$ decrease in maternal mortality, it is likely that its efficacy could be improved.

Unlike the Pneumatic Anti-shock Garment (PASG), the NASG has no manometer to determine how much pressure is being exerted and whether the device has been applied too loosely (resulting in ineffective vascular compression) or too tightly (resulting in ischemia or compartment syndrome). Since the NASG does not have a pressure gauge, it is not known how application pressure varies (i.e. between a strong applier and a weaker applier), although providers are trained to test the tightness of their application by lifting up a part of an applied segment with one finger and letting it 'snap' back into place to ensure a level of tightness. This is a very unreliable method and the variable pressure of application would 

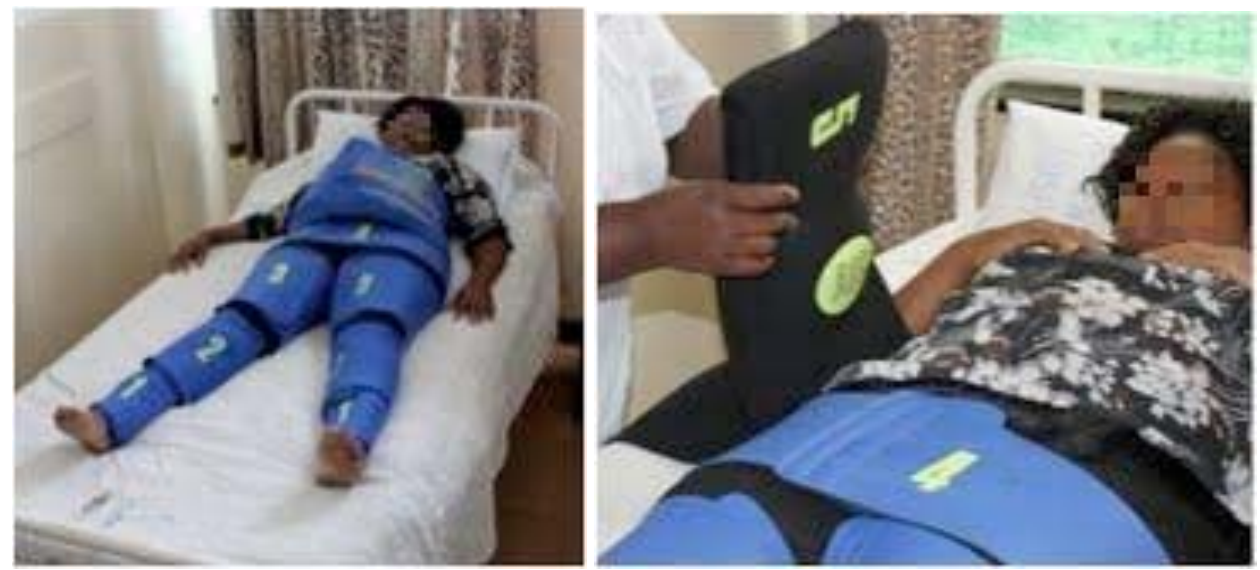

Fig. (1). Photographs of a Zimbabwean woman with the NASG fully applied (left) and of the placement of the abdominal segment with the uterine compression ball (right).

likely result in variable efficacy. It is also unknown whether the pressure varies in patients with different body mass indices (BMIs). While these may both seem intuitive, there is no specific advice from the manufacturer of the device, who simply instructs that the device be applied "as tightly as possible" [10]. Examining different pressures of application, and different BMIs, is an important step towards optimizing NASG efficacy.

The objective of this pilot study was to use non-invasive methods to characterize the external abdominal pressure generated by NASG application and to elucidate how BMI and applier strength affect outcomes.

\section{MATERIALS AND METHODOLOGY}

The NASG is comprised of 5 articulated segments, the abdominal segment contains a foam ball for extra compression on the abdomen [11]. At least two main theoretical mechanisms of action underlie the effectiveness of the NASG. The first involves the shunting of blood flow from the lower extremities and pelvis back to the central circulation in order to improve perfusion of vital organs. The second involves the pressure exerted by the abdominal compression ball onto the uterus and surrounding vasculature in order to decrease blood flow to the pelvis and diminish uterine blood loss. Hauswald et al. have shown a decrease in blood flow below the distal aorta with placement of the NASG [12] and our group has recently demonstrated that NASG application increases the resistive index in the internal iliac arteries in postpartum patients [13].

Approval for this study was obtained from the University of California, San Francisco (UCSF) and the PATH (Program for Appropriate Technology in Health), Institutional Review Boards. Participation was voluntary and all study subjects participated in an informed consent process.

The study was conducted at the UCSF Moffitt Hospital on the Labor and Delivery ward. The study was done in two phases with 10 participants in each phase. The initial phase measured the pressure generated by the NASG on the external abdominal wall with a single experienced NASG applier. The second phase investigated the effects of applier strength and patient BMI on the pressure generated with NASG application.

\section{Phase One: Pressure with Single Applier}

Ten healthy, female, non-pregnant, non-postpartum volunteers were recruited and consented to the study. Demographics including age and gravidity were recorded. A Koala Intra-Uterine Pressure Catheter (IUPC) was placed $2 \mathrm{~cm}$ below the umbilicus and secured in place. The manometer was then calibrated to zero. Prior to the start of the study, the IUPC was tested against a sphygmomanometer to verify the accuracy of the IUPC measurement. Vital signs including pulse, respirations, blood pressure, and oxygen saturation as well as external abdominal pressure were recorded at 5minute intervals with serial application of the NASG as follows:

1. Baseline (no NASG)

2. Immediately after full application of the NASG

3. Five minutes after full application of NASG

4. Immediately after complete removal of NASG

5. Five minutes after complete removal of NASG

\section{Phase Two: BMI and Applier Strength Effects}

In order to better understand the effect of body habitus on NASG parameters, volunteers with varied BMI were recruited. A total of 10 non-pregnant female volunteers were recruited; 3 underweight (BMI <19), 4 normal weight (BMI 19-25) and 3 overweight/obese (BMI >25). While some of these volunteers were the same as used in Phase 1 , Phase 2 was conducted a few days later to ensure there was no residual effect from Phase 1. We also investigated the effect of NASG application by persons of different strengths by recruiting "strong" and "weak" appliers based on previously self reported physical strength and body habitus. Each applier was asked to apply the device using all of their strength, the manufacturer's sole recommendation for application, on the 10 patients described above. A minimum interval time of 20 minutes was used between application on the same individual by the "strong" and "weak" appliers. Demographic data and any side effects experienced by the patients were documented. Pressure and vital signs were recorded at the same intervals as described for phase one above.

Data were entered into Microsoft Excel for Mac 2008 (Microsoft Corporation, WA) and analyzed using SAS ver- 
sion 9.2 (SAS Inc, Cary NCO). Descriptive statistics were reported for both phases. In both phases, the $\mathrm{p}$ values for comparing means by applier, BMI group and time were computed using a parametric repeated measure analysis of variance model. The model was modified so times where the pressure was zero in all subjects did not contribute to and distort the variance estimates. The $\mathrm{p}$ values for pair wise mean comparisons were computed under this model using the Fisher least significant difference (LSD) criterion. Correlations were assessed between continuous variables, such as pressure versus BMI, using the non-parametric Spearman correlation coefficient since the relationship was not necessarily intrinsically linear. We have considered results of $\mathrm{p}=0.05$ or less to be statistically significant.

\section{RESULTS}

\section{Phase One: Pressure with Single Applier}

Ten healthy non-pregnant, non-postpartum patients were recruited; average age, BMI, gravidity and parity are documented in Table $\mathbf{1}$. The majority of patients were nulligravid $(9 / 10)$, while 1 woman had previously delivered 2 children. None of the patients reported any side effects during NASG application. Vital signs remained stable throughout with no significant changes. Mean abdominal pressures were low at baseline, $-1.4 \mathrm{mmHg}$, SEM 1.38, with a significant rise upon full application after 5 minutes, $66.6 \mathrm{mmHg}$, SEM 3.56, and a rapid return to near baseline levels upon complete removal, $-1.9 \mathrm{mmHg}$, SEM 1.21 (Fig. 2). Pressure after full application of the NASG was significantly greater than pressure immediately before application $(\mathrm{p}<0.001)$ and upon removal $(\mathrm{p}<0.001)$.

Table 1. Demographic Data for the Pressure Study - Phase 1

\begin{tabular}{|c|c|}
\hline & $\begin{array}{c}\text { Phase One } \\
\text { Mean (Range) } \mathbf{n}=\mathbf{1 0}\end{array}$ \\
\hline \hline Age (years) & $31(22-42)$ \\
\hline BMI & $22(19-26)$ \\
Underweight & $19(\mathrm{n}=1)$ \\
Normal Weight & $22(20-24)(\mathrm{n}=8)$ \\
Overweight & $26(\mathrm{n}=1)$ \\
\hline Gravidity & $0.2(0-2)$ \\
\hline Parity & $0.2(0-2)$ \\
\hline
\end{tabular}

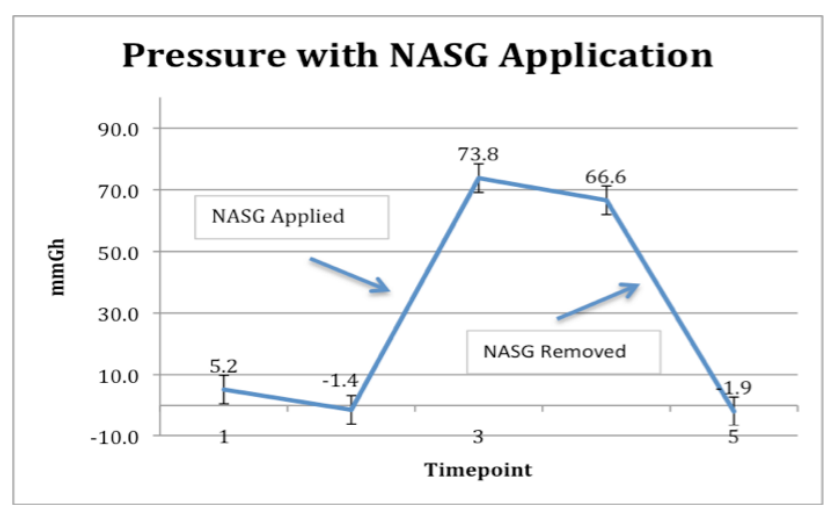

Fig. (2). The effect of NASG on mean external abdominal wall pressure $(n=10)$.

\section{Phase Two: BMI and Applier Strength Effects}

Ten healthy non-pregnant, non-postpartum volunteers with varied BMIs were evaluated (see Table 2). Average age was 33 years. The majority of women were nulligravid $(8 / 10)$ and the other two patients had delivered 2 children each. For these 10 patients, mean BMI by BMI category were as follows: 3 underweight (mean BMI 18.3), 4 normal weight, (mean BMI 21.0) and 3 overweight/obese (mean BMI 30.3).

For all participants, application of the NASG resulted in a significant increase in pressure. There were no significant changes in vital signs with application of the NASG and no reported side effects. There was a high degree of correlation between the pressure generated immediately after application and after 5 minutes with both strong appliers (Spearman correlation $=0.963, \mathrm{p}=0.001$ ) and weak appliers (Spearman correlation $=0.854, \mathrm{p}=0.002$ ); therefore the means of time-points 2 and 3 are reported in Fig. (3). Application of the NASG by the strong applier vs. the weak applier for all three BMI groups resulted in higher mean pressure (Fig. 3). The difference between the pressure generated by a strong applier in an underweight patient $(73.2 \mathrm{mmHg})$ and a weak applier in an overweight patient $(35.7 \mathrm{mmHg})$ was statistically significant (Wilcoxon rank sum test; $\mathrm{p}=0.051$ ).

Table 2. Demographic Data for the Pressure Study - Phase 2

\begin{tabular}{|c|c|}
\hline & $\begin{array}{c}\text { Phase 2 } \\
\text { Mean (Range) } \mathbf{n}=\mathbf{1 0}\end{array}$ \\
\hline \hline Age (years) & $33(23-40)$ \\
\hline BMI & $23(18-33)$ \\
Underweight & $18.3(18-19)(\mathrm{n}=3)$ \\
Normal Weight & $21.0(20-22)(\mathrm{n}=4)$ \\
Overweight/obese & $30.3(28-33)(\mathrm{n}=3)$ \\
\hline Gravidity & $0.5(0-3)$ \\
\hline Parity & $0.4(0-2)$ \\
\hline
\end{tabular}

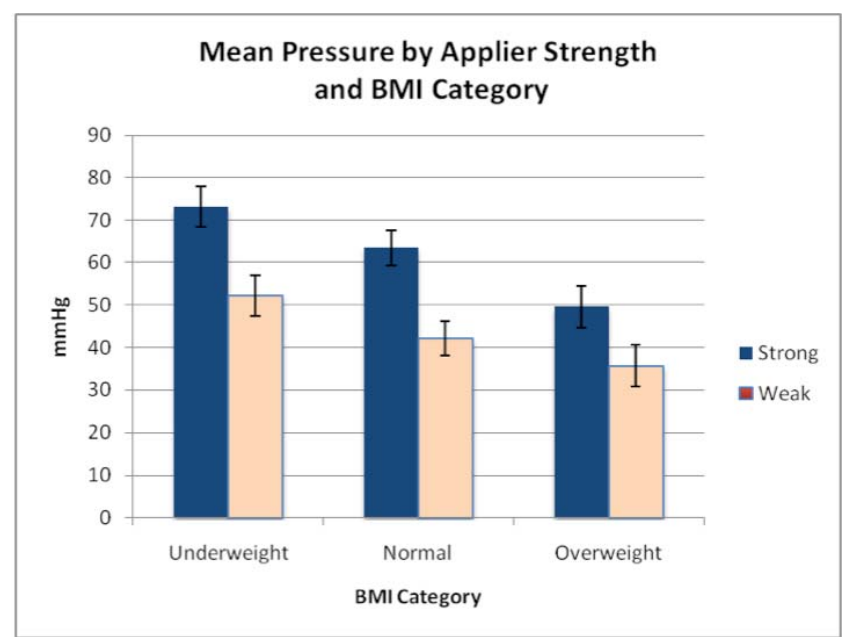

Fig. (3). Mean pressure during NASG application by strong and weak appliers for each BMI category, with standard error bars $(\mathrm{n}=10)$. 
There was an inverse relationship between pressure and BMI, which was statistically significant for the strong applier (Spearman correlation $=-0.905, \mathrm{p}<0.001$ ), but not significant for the weak applier (Spearman correlation $=-0.232$, $\mathrm{p}=0.518$ ). Placement of the NASG abdominal segment for 5 minutes resulted in a rapid rise in mean pressure in both the strong and weak applier groups $(62.0$ vs $43.3 \mathrm{mmHg}$, SEM 2.64) (see Fig. 4). The mean pressure generated by the strong applier was significantly greater than that generated by the weak applier (ANOVA; $\mathrm{p}<0.001$ ). Pressure rapidly returned to baseline after removal of the NASG. Vital signs did not change significantly with application of the NASG.

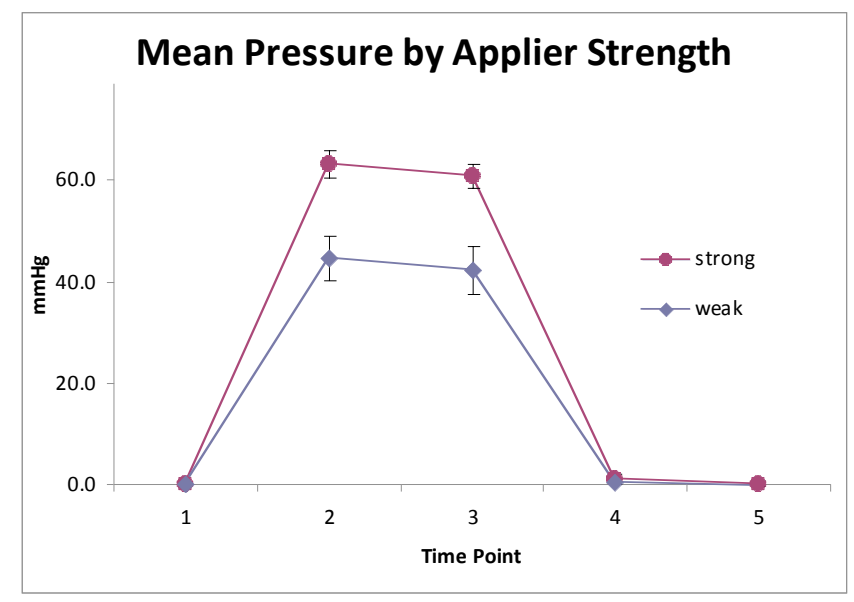

Fig. (4). The effect of applier strength on mean pressure with NASG application $(n=10)$.

\section{DISCUSSION}

Both NASG efficacy and side effects likely depend on the circumferential compression of vessels from pressure exerted by the device, therefore, understanding how pressure varies in individual applications is critically important. This study demonstrates that external abdominal pressure increases with NASG application, and that the pressure generated varies with applier strength and BMI. The NASG has no pressure gauge or mechanism to inform appliers that the appropriate pressure is being exerted. This initial evaluation of how pressure varies within patients and appliers provides critically important baseline information that can inform development of means to ensure that appropriate pressure is being exerted with each application of the NASG.

Severe postpartum hemorrhage affects a diverse cohort of over 100,000 women annually throughout the world [2, 14]. The body habitus of a woman affected in rural India may be very different than a woman in peri-urban Nigeria or in the Peruvian highlands. It is therefore important to consider the height, weight, BMI and habitus of prospective patients. Similarly, health care providers vary in size and strength; the maximal pressure generated by a stronger applier is likely to be significantly different than a weaker applier. It may be that individualized training methods could better standardize the amount of pressure generated. Alternatively, it may be beneficial to develop a simple pressure gauge, or other means of assuring appropriate placement in patients with different body habitus. For now, this study will serve to influence training in the use of the NASG by providing a recommendation that weaker providers should be conscious that their application needs to be as strong as possible, that they position themselves adjacent from the patient in order to use their body weight to ensure a snugger fit, and to check each segment for a clear 'snapping' sound. If there is no 'snap,' indicating that the material is not pulled tightly enough, they should reapply the section or seek assistance from a stronger member of staff.

The ideal amount of pressure that should be generated with NASG application has not been demonstrated. Further, the current sole manufacturer of the device gives no information on whether one should apply more or less pressure depending on the size of the patient. Studies to more fully elucidate the impact of the NASG on intra-abdominal vascular flow would clarify the relationship between pressure applied and perfusion of intra-abdominal organs (e.g. uterus, intestines, kidney). It would also be important to correlate the pressure exerted by the NASG with the cardiovascular impact on the central circulation, namely central venous pressure, systemic vascular resistance and cardiac output.

An inverse relationship between pressure and BMI was observed in this study. This effect was more significant in the strong appliers, than in the weak appliers. There are two factors that may be important to consider in this finding. One is that in very small patients, it is necessary to wrap the NASG more securely around the patient to overcome the size constraints of the device, which could be difficult for a small, weak applier. The second is that fatigue could have played a role, particularly in the non-conditioned, weaker applier. Training should emphasize the importance of securing the device as firmly as possible and seeking help from another member of staff if fatigued.

In order to use non-invasive means of study, external pressure was measured directly between the uterine compression ball and the abdominal wall. This is likely to overestimate intra-abdominal pressure and the effect of body habitus may also not be accurate. The next step for studying pressure would be to further assess these findings using a more accurate approximation of intra-abdominal pressure, such as a transrectal or transurethral pressure gauge. Once these measurements are established, perhaps an approximation by correlation or formula can be developed to transfer to measurement of external pressures.

This study was limited by a small sample size and the use of non-pregnant healthy female volunteers. As Phase I only included only one underweight and one overweight woman, and both used a small sample size, future studies should expand the sample size and recruit a larger number of participants with different body habitus, including height, weight, BMI, abdominal circumference and ethnicity. It may also be useful to conduct this study in a postpartum cohort, as the physiology of pregnancy and the puerperium may result in some important differences in pressure outcomes. Similarly, the effect of applier size and strength should be further explored by evaluating a more diverse cohort of appliers with varied height, weight, strength, gender and ethnicity, and using a standardized method of strength testing to evaluate applier strength and its correlation to pressure.

Understanding how pressure, vascular flow and cardiovascular function are related in a diverse cohort of patients and health care providers is an important step towards opti- 
mizing NASG application. Studies have evaluated blood flow in the distal aorta with application of both the PASG and NASG $[12,13,15]$, demonstrating that flow is substantially diminished by both, but more so with the PASG. However, this does not necessarily mean that blood flow to the uterus is also decreased more so by the PASG. The NASG has a lower abdominal compression ball that may affect the blood flow to the uterus independently. Blood flow to the lower extremities may be preserved more so with the NASG than the PASG. For example, lower extremity ischemia and compartment syndrome have been reported with the PASG, but not with the NASG [16-19]. Future studies should endeavor to define these relationships and to more clearly elucidate the individual characteristics that affect NASG application. If significant variation persists in future studies, modification of NASG design (e.g. simple pressure gauge) or improved training protocols may result in improved individual application. The goal of these future studies would be to ensure that each placement of the NASG will result in optimal results: immediate diminished uterine bleeding, resuscitation from shock, and survival without adverse effects. Achieving this objective may improve obstetric shock management and reduce deaths from obstetric hemorrhage, the world's leading cause of maternal mortality.

\section{TRIAL REGISTRATION}

UCSF CHR approval \# 10-00506.

\section{CONFLICT OF INTEREST}

The authors declare no conflict of interest.

\section{ACKNOWLEDGEMENTS}

The study was funded by the Program for Appropriate Technology in Health (PATH).

\section{REFERENCES}

[1] WHO. Trends in Maternal Mortality: 1990 to 2008: Estimates developed by WHO, UNICEF, UNFPA and The World Bank. Geneva 2010.

[2] Hogan MC, Foreman KJ, Naghavi M, et al. Maternal mortality for 181 countries, 1980-2008: a systematic analysis of progress towards Millennium Development Goal 5. Lancet 2010; 375(9726):1609-23.
[3] Gabrysch S, Campbell OM. Still too far to walk: literature review of the determinants of delivery service use. BMC Pregnancy Childbirth 2009; 9: 34 .

[4] Thaddeus S, Maine D. Too far to walk: maternal mortality in context. Newsl Womens Glob Netw Reprod Rights 1991; 36: 22-4.

[5] Miller S. Anti-Shock Garments: Non-pneumatic (NASG) and pneumatic.: California maternal quality care collaborative; Available from: www.cmqcc.org/resources/636/download

[6] Miller S, Martin H, Morris J. Anti-shock garment in postpartum haemorrhage. Best Pract Res Clin Obstet Gynaecol 2008; 22(6): 1057-74.

[7] Miller S, Hamza S, Bray EH, et al. First aid for obstetric haemorrhage: the pilot study of the non-pneumatic anti-shock garment in Egypt. Br J Obstet Gyneeol 2006; 113(4): 424-9.

[8] Miller S, Turan JM, Dau K, et al. Use of the non-pneumatic antishock garment (NASG) to reduce blood loss and time to recovery from shock for women with obstetric haemorrhage in Egypt. Glob Public Health 2007; 2(2): 110-24.

[9] Miller S, Ojengbede O, Turan J, Morhason-Bello I, Martin H, Nsima D. A comparative study of the non-pneumatic anti-shock garment for the treatment of obstetric hemorrhage in Nigeria. Int J Gynaecol Obstet 2009; 107(2): 121-5.

[10] Zoex. How do you apply the ZOEX NIASG. [Video] [updated 924-10]; Available from: http://www.zoexniasg.com/video/

[11] Miller S, Ojengbede A, Turan J, Ojengbede O, Butrick E, Hensleigh P. Anti-shock garments for obstetric hemorrhage. Curr Women's Health Rev 2007; 3(3): 3-11.

[12] Hauswald M, Williamson MR, Baty GM, Kerr NL, Edgar-Mied VL. Use of an improvised pneumatic anti-shock garment and a non-pneumatic anti-shock garment to control pelvic blood flow. Int J Emerg Med 2010; 3(3): 173-5.

[13] Felicia Lester, Amy Stenson, Carinne Meyer, Jessica Morris, Juan Vargas, Suellen Miller. The Impact of the Non-Pneumatic AntiShock Garment on Pelvic Blood Flow in [Healthy Post-Partum. Am J Obstet Gynecol (accepted in press).

[14] Khan KS, Wojdyla D, Say L, Gulmezoglu AM, Van Look PF. WHO analysis of causes of maternal death: a systematic review. Lancet 2006; 367(9516): 1066-74.

[15] Hauswald M, Greene ER. Regional blood flow after pneumatic anti-shock garment inflation. Prehosp Emerg Care 2003; 7(2): 225 8.

[16] Aprahamian C, Gessert G, Bandyk DF, Sell L, Stiehl J, Olson DW. MAST-associated compartment syndrome (MACS): a review. J Trauma 1989; 29(5): 549-55.

[17] Brotman S, Browner BD, Cox EF. MAS trousers improperly applied causing a compartment syndrome in lower-extremity trauma. J Trauma 1982; 22(7): 598-9.

[18] Randall P, Banks J, Little RA. Medical (military) anti-shock trousers--a short review. Arch Emerg Med 1984; 1(1): 39-51.

[19] Williams TM, Knopp R, Ellyson JH. Compartment syndrome after anti-shock trouser use without lower-extremity trauma. J Trauma 1982; 22(7): 595-7. 